\title{
A Pragmatic Approach for EEG-based Affect Classification
}

\author{
Anju Mishra" ${ }^{* 1}$ Archana Singh ${ }^{2}$, Amit Ujlayan ${ }^{3}$
}

\begin{abstract}
Submitted: 06/07/2021 Accepted : 20/10/2021
Abstract: Mapping human cognition into automated analysis is the key area of research due to its fascinating applications in almost every area of developing artificially intelligent machines. The best way to understand the functioning of brain is to study electroencephalogram (EEG) patterns, therefore, a lot of research has been directed towards studying EEG signals. Since, EEG recordings are subject dependent and exhibit variations due to external influences or type of recording instruments, it is hard to develop a generalized affect categorization system that can provide robust affect labelling to the EEG patterns. To overcome this, proposed work presents a novel general framework for affect-based cognitive analysis. The proposed system involves following steps: pre-processing, feature selection, Generalized Procrustes Analysis (GPA) step to reduce the inter-class and intra-class variance and finally, the processed pattern is passed on to a trained classifier for classifying the pattern into appropriate affect categories. The presented approach has been tested on single as well as multiple subjects' EEG data taken from two different datasets, Database for Emotion Analysis using Physiological signals (DEAP) and SJTU Emotion EEG Dataset (SEED) and performance of popular classifiers are assessed. The experimental results suggest that SVM classifier is the best among the selected ones for classifying single as well as mixed subjects' data.
\end{abstract}

Keywords: Affective analysis, Affect-based cognition, EEG signals, GPA analysis, Video tagging

This is an open access article under the CC BY-SA 4.0 license. (https://creativecommons.org/licenses/by-sa/4.0/)

\section{Introduction}

Emotion analysis using physiological signals is a key area of current research which involves analysing human emotions from electrical impulses generated from human body because of electrochemical reactions pertaining to some tasks. These impulses are generated from different parts of body and accordingly categorized into different categories viz. EEG, ECG, EMG etc. Out of these, ECG and EEG signals are preferable and in literature, a lot of experimental procedures have been applied on these signals to detect and identify variety of modalities, be it the detection of different types of disorders or emotions or motor imagery for task identification etc. [32.33.24]. Since, the phenomenon of intelligence is associated with human brain, majority of intelligent systems have taken inspiration from functioning of human brain cognition by studying physiology of brain. Brain cognition is essentially the processing of external stimuli for creating new knowledge or deciding what action or response is to be given. Various types of regression or classification systems have been proposed which made use of EEG pattern recognition, be it the neuro-prosthetics for helping differently abled persons or various types of brain disease detection systems [24,25,26,27,28,29,30,31]. As, EEG patterns are low amplitude signals recorded from over the scalp, this requires a lot of preprocessing and specialized techniques to classify them using soft computing techniques. Out of these signals, a lot of experiments

${ }^{1 *}$ Amity School of Engineering \& Technology, Amity University Uttar Pradesh, Noida, India, ORCID ID: 0000-0003-4485-1520

${ }^{2}$ Amity School of Engineering \& Technology, Amity University Uttar

Pradesh, Noida, India, ORCID ID: 0000-0001-5365-6351

${ }^{3}$ School of Vocational Studies and Applied Sciences, Gautam Buddha

University, Greater Noida, India, ORCID ID: 0000-0002-6045-8385

*Corresponding Author Email: anju.iitd@gmail.com have been applied on EEG and ECG to detect and a variety of modalities [3,4,8-16, 18-23,40-41].

The major problems that arise while recording of EEG signals and which contribute to bringing down the performance of a classifier are:

1. These are low-voltage signals with very low amplitude measured in $\mu$-volts.

2. High risk of noise arising from breathing and muscle activities etc. which corrupts the signals while recording.

3. Correct recording of signals requires substantial user attention and focus while performing the task.

4. Signals are subject specific and therefore, hard to generalize and depend upon current and previous states of mind.

A lot of work has already been done to recognize human emotions from facial expressions, speech, text, and a mixture of these characteristics [15]. In [19] a systematic review is presented which compares well-known methods used in pattern recognition system. A novel Multi-modal Correlated Network for emotion recognition was proposed in [24]. The authors have utilized a fusion of audio and visual information for emotion recognition. The audio features were employed as images called as Mel-spectrograms and visual frames were fed to CNN and LSTM for extracting features and then applied feature fusion method to classify emotions. A variety of classifiers such as convolutional neural networks (CNNs), hidden Markov models (HMMs), re-current neural networks (RNNs), long-short term memory (LSTM) classifiers, decision tree and support vector machine (SVM) classifiers, have been used in literature by different authors for classification of emotions from facial expression, speech, images and their mix $[24,25,26,27,28,29,30,31,35,36,37,38]$. Since, all these characteristics of humans can be overshadowed or con-trolled by 
oneself due to the presence of a variety of environmental or personal factors, sometimes it becomes hard to exactly guess the right emotion of a person for further assessment. Therefore, emotion recognition from physiological quantities such as EEG comes into picture, which are the direct responses of human brain to emotional stimuli. A wide variety of approaches have been tested for identification of emotions from EEG signals. In [3], a thorough survey of existing research for emotion recognition using EEG signals have been presented. The survey focused on different aspects of an EEG based emotion recognition system involving sub-jects, types of features extracted, and types of classifiers used. In [4] authors have claimed the superiority of Dis-criminative Graph regularized Extreme Learning Machine with differential entropy features for emotion classification on DEAP and SEED datasets. In another study [13] a review on feature extraction methods per-formed on EEG data for emotion recognition was presented. The review was based on 33 studies and com-pared the performance of system with respect to the choice of feature selection method and choice of electrode locations. In [14] emotional states are recognized from EEG signals recorded for twenty-one healthy sub-jects with fourteen electrodes while showing the subjects images. The authors made use of Hjorth parameters (activity, mobility, and complexity) for selecting optimal EEG features. These features were then processed by a variety of classifiers including support vector machine, k-nearest neighbour, linear discriminant analysis, Naive Bayes, random forest, deep learning, and a combination of four ensembles methods bagging, boosting, stacking, and voting were employed for emotion classification. The research in the domain has been extended in multiple directions and researchers added more dimensions to the problem of emotion recognition using EEG signals. Recently researchers have tried to explore the domain adaptation techniques so that the classifier can perform efficiently on any type of EEG dataset, thereby enhancing the generalization capability of the classifiers. In this direction, in [22] A subjectindependent classifier that is trained using transfer learning or domain adaptation techniques have been proposed. The work focused on cross-dataset learning performed on two popular datasets: DEAP and SEED and suggested that domain adaptation techniques can improve the classification accuracy on both datasets by reducing the inter-subject variance.

To handle the complexity of EEG patterns, this work proposes a novel generalized approach for classification of human affective states using EEG patterns. The proposed work considers following research questions and tries to answer them:

1. Can the accuracy of a classifiers be improved despite of the above-mentioned drawbacks with EEG recordings?

2. Since, computational cost is a major challenge to cope up with while developing real-time systems using EEG. Can the dimensionality of EEG data be reduced without affecting the performance of classifier in question?

3. Can a classifier be made to generalize enough to work for multiple subjects' data?

To assess the effectiveness of proposed work, the method was tested on four popular classifiers used in literature. The presented work applies Generalized Procrustes Analysis (GPA) on preprocessed EEG patterns which helps in registering channel data recorded while watching a video with other EEG patterns so that they become more comparable. After this, data are passed on to the classifier for training and testing. The basic steps of processing in the proposed approach are illustrated in Fig. 1.

\subsection{Generalized Procrustes Analysis (GPA)}

Generalized Procrustes analysis is a statistical method, which was traditionally introduced to analyse the free-choice profiling results for surveying where respondents were free to choose their language or words for describing products. It has been used to analyse results of interviews, surveys, panels, and even for comparing shapes of objects. This is a type of statistical multivariate analysis technique which works by superimposing shapes in a series using Procrustes. The process of superimposition involves translation, rotation, and scaling of shape landmark points to match them with a reference shape. The main objective of GPA technique is to find the optimal alignment of group of sample shapes by selecting the minimum distance of group with mean shape. The method starts by estimating scaling factor by utilizing a weight factor which compensates between the reference or mean shape and a group of other shapes. The method involves $n$ points in 2D space and calculating centroids of shape landmark points for each shape and then translate them to the landmark points of reference shape called as origin [5,6,7]. The $n$ landmark points in 2D space are represented as:

$\left\{\left(x_{1}, y_{1}\right),\left(x_{2}, y_{2}\right),\left(x_{3}, y_{3}\right), \ldots \ldots,\left(x_{n}, y_{\mathrm{n}}\right)\right\}$

and

$X_{C}=\frac{x_{1}+x_{2}+x_{3}+\cdots \ldots+x_{\mathrm{n}}}{n}$

and

$Y_{c}=\frac{y_{1}+y_{2}+y_{3}+\cdots . .+y_{\mathrm{n}}}{n}$

Where, Xc and Yc are the coordinates of centroid. All the points are then translated to the origin as:

$f\left(x_{i}, y_{i}\right)=\left(x_{\mathrm{i}}-X_{c}, y_{\mathrm{i}}-Y_{c}\right)$

Where, $\forall i: i \in(1,2,3, \ldots \ldots, n)$. Next, all the points are scaled with respect to origin as:

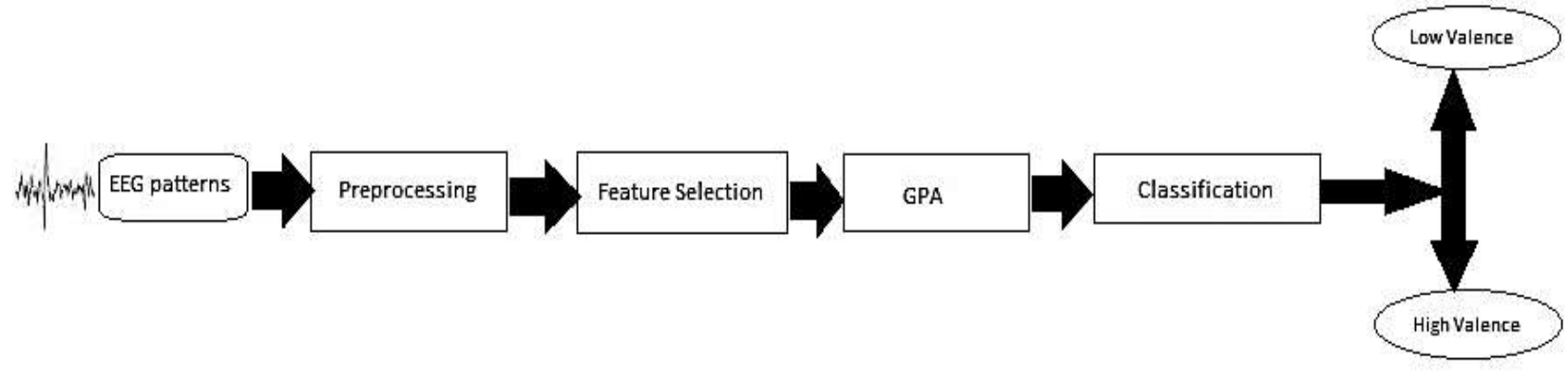

Fig. 1. General processing steps 


$$
\left\{\begin{array}{c}
\left(\frac{x 1-X c}{s}, \frac{y_{1}-Y_{c}}{s}\right),\left(\frac{x_{2}-X_{c}}{s}, \frac{y_{2}-Y_{c}}{s}\right), \ldots . \\
,\left(\frac{x_{n}-X_{c}}{s}, \frac{y_{n}-Y_{c}}{s}\right)
\end{array}\right\}
$$

here, $\mathrm{s}$ is the size of centroid of shape and calculated as:

$$
s=\left\{\begin{array}{c}
\left(x_{1}-X_{c}\right)^{2}+\left(y_{1}-Y_{c}\right)^{2}+ \\
\left(x_{2}-X_{c}\right)^{2}+\left(y_{2}-Y_{c}\right)^{2}+\cdots \\
+\left(x_{n}-X_{c}\right)^{2}+\left(y_{n} Y_{c}\right)^{2}
\end{array}\right\}^{\frac{1}{2}}
$$

Finally, rotation metric is calculated in terms of $\theta$ angle by keeping the reference shape fixed and rotating the other shape with respect to it so that the distance between them is minimized.

\subsection{Dimensionality Reduction}

Dimensionality reduction refers to the process of reducing the number of random variables used in the data. This process maps the data from high dimensional space to a lower dimensional feature space so that the cost of computation and storage can be cut down or reduced. A variety of methods are available for this such as low variance filter, missing value ratio, high correlation filter principal component analysis (PCA), backward feature elimination etc $[12,13,14,18,19,23]$. Dimensionality reduction can be performed in two ways: selecting relevant features called feature selection and extracting relevant features from data that can effectively represent data, this approach is called feature extraction. In our work dimensionality reduction was achieved by employing second approach i.e., extracting statistical features from data. Nine different statistical features were extracted from each EEG channel data viz., mean, median, standard deviation, variance, min, max, range, skewness, and kurtosis [39]. A thorough description of dimensionality reduction process used in the proposed work is outlined in section III.

\section{Experimental Data and Procedure}

It is an established fact that EEG patterns are subject specific, therefore, to device a generalized method for their classification, the proposed work is divided into three parts:

A. Classifying Individual Subject's EEG patterns Separately

B. Classifying Multiple Subjects' EEG patterns

C. Classifying Mixed EEG patterns from different Datasets

The first category involves experiments on single subject data taken from each of DEAP and SEED datasets respectively. The second category of experiments focus on analyzing performance of classifier on multiple subjects' EEG data taken from a single dataset (all subject either belong to DEAP or SEED). In the third category of proposed work, experiments are conducted on mix of EEG data obtained from both DEAP and SEED datasets to show the performance of classifier in a general case (when data is recorded by different equipment by different researchers). The general experimental procedure followed for each of the experimental category starts by first taking recorded EEG data and preprocess it. This step is crucial as it helps in removing or minimizing the noise present in the recorded EEG signals as well as conditioning the signal. After this, features from EEG patterns were extracted by classifier and GPA was applied and then the data was classified into appropriate affect category. Typical steps of processing are illustrated in Fig. 1.

\subsection{Experimental Data}

This work uses two different datasets namely Database for Emotion Analysis using Physiological signals (DEAP) [1] and
SJTU Emotion EEG Dataset (SEED) [2]. Both datasets are multimodal in nature. The reason for taking two datasets and their mix was twofold, to evaluate the performance of proposed method on cross-subject as well as on cross-dataset subjects' data. This was necessary to prove the specificity as well as generality of the proposed method.

\section{DEAP Dataset}

DEAP dataset is a multimodal dataset recorded by Koelstra et al. for analysing emotional modalities from EEG patterns. The dataset contains recorded EEG and peripheral physiological patterns of thirty-two subjects while showing them music videos of oneminute duration. Forty different videos were used for this purpose. All participants were asked to rate these videos according to predefined rating scale as per the level of arousal, valence, like/dislike, dominance, and familiarity. All recordings were done with a sampling rate of $512 \mathrm{~Hz}$ by using 32 active $\mathrm{AgCl}$ electrodes.

\section{SEED Dataset}

SEED is also a multimodal dataset recorded with fifteen subjects when they were watching movie clips [2]. Fifteen different movie clips were selected for three emotional modalities: negative, positive, and neutral. Each experiment included fifteen trials and each experiment was repeated three times for each subject. EEG recordings were taken with 62 electrodes as per international 1020 system.

\subsection{Experimental Method}

The experiments were carried out on EEG recordings of each of the DEAP and SEED datasets. For simplicity only one emotional modality 'valance' (responses recorded on a continuous scale of 1 to 9) of DEAP dataset was chosen and its ratings were converted to two discrete levels- high valence (HL) if the rating falls in the range 4.5 to 9 and low valence (LV) if its value is less than 4.5. For simplicity of experiments pre-processed EEG data was taken from both DEAP and SEED datasets. All experiments were performed in MATLAB environment on a single CPU.

\section{A. Classifying Single Subject EEG Data}

The pre-processed EEG signal data of one subject was considered from each of the datasets for experimentation. The one subject preprocessed DEAP data was already down sampled to $128 \mathrm{~Hz}$ and after that EOG removal, filtering, and segmentation steps were already performed. The data is a three-dimensional array where first dimension corresponds to number of videos shown to each subject, second dimension specifies number of channels and the last indicates number of points. In our experiments, we used thirty EEG and eight peripheral signals having a total of forty labels corresponding to 'valence' modality containing two discrete values: HV (high valence) and LV (low valence) which specify 'Happy or Excited' and 'Sad or Depressed' emotional categories respectively. Next, the EEG data corresponding to labels HV and LV were separated. Next, data reduction is performed similar to that mentioned in [39], first dividing the data into six batches of around 1330 points and then statistical features were extracted from these segments of EEG data. Here, we made use of nine statistical features viz., mean, median, max, min, standard deviation, variance, range, skewness, and kurtosis. Therefore, we have a total of ninety features per channel plus nine additional features calculated for full channel data i.e., for all 8064 points thereby making a total of ninety-nine features per channel. After this, the data was divided into two groups as per their labels, and GPA was applied on both the groups separately. This step ensures 
that the signatures of different EEG signals corresponding to similar affect category are registered with each other as closely as possible. Afterwards, the data from each affect category was divided into training and testing sets. Here, we divided the data in a ratio of 80:20 for training and testing respectively. The data along with corresponding labels were passed on to the classifier for classification. The motive behind this is to select the appropriate classifier for the proposed model. The training data is used to train four different classifiers and the performance of these classifiers are analysed using test data.

Table 1. Accuracy (\%) for Single Subject Data (Valence Attribute)

\begin{tabular}{|l|c|c|c|c|}
\cline { 2 - 5 } \multicolumn{1}{c|}{} & \multicolumn{4}{c|}{ Dataset } \\
\cline { 2 - 5 } \multicolumn{1}{c|}{} & \multicolumn{2}{c|}{ DEAP } & \multicolumn{2}{c|}{ SEED } \\
\hline Classifier & $\begin{array}{c}\text { Proposed } \\
\text { Method }\end{array}$ & F-score & $\begin{array}{c}\text { Proposed } \\
\text { Method }\end{array}$ & F-score \\
\hline $\begin{array}{l}\text { Support Vector } \\
\text { Machine }\end{array}$ & 95.0 & 0.9299 & 92.5 & 0.9299 \\
\hline K-Nearest Neighbor & 87.5 & 0.8873 & 50.0 & 0.5027 \\
\hline Decision Tree & 87.5 & 0.8873 & 87.5 & 0.8873 \\
\hline Naïve Bayes & 87.5 & 0.8873 & 92.5 & 0.9299 \\
\hline
\end{tabular}

All the results are summarized in Table 1 under DEAP data category. Similar course of action was performed for SEED dataset. We chose a single subject's preprocessed EEG recordings and repeated the experiments on this. The size of SEED data for a single subject was $15 \times 62 \times 37000$ (i.e., number of video clips $\mathrm{x}$ number of channels $\mathrm{x}$ number of points). The labels in seed data correspond to three classes negative, positive, and neutral. For uniformity across experiments, we only considered positive and negative labels and corresponding data. The data was divided into two groups according to two labels and GPA was applied on that, then eighty percent of the processed data was used for training the classifiers. The classification performances of chosen classifiers were evaluated on test data.

Table 2. Accuracy (\%) for Multiple Subjects on Valence Attribute (all subjects taken from same dataset)

\begin{tabular}{|l|c|c|}
\cline { 2 - 3 } \multicolumn{1}{c|}{} & \multicolumn{2}{c|}{ Dataset } \\
\hline Classifier & DEAP & SEED \\
\hline $\begin{array}{l}\text { Support Vector Machine } \\
\text { (with Poly kernel degree=2) }\end{array}$ & 95.0 & 84.0 \\
\hline K-Nearest Neighbor & 87.5 & 60.0 \\
\hline Decision Tree & 87.5 & 87.5 \\
\hline Naïve Bayes & 87.5 & 87.5 \\
\hline
\end{tabular}

\section{B. Classifying Multiple Subjects' Data}

Experiments were repeated for EEG data of multiple subjects of DEAP and SEED datasets. This step involved combining preprocessed data of multiple subjects into one array for each of the datasets. For DEAP dataset, we merge EEG recordings of ten subjects together. The labels were also merged into an array. Similar methodology was followed for this category, first performing dimensionality reduction step followed by applying GPA and then supplying data to classifiers and recording their performance. The results for this category of experiments are summarized in Table 2. Again, the performance of SVM classifier is found to be more consistent for classifying this category's data.

\section{Classifying Mixed data from both datasets}

In proposed work, we also performed the affect classification on
EEG data taken from both DEAP and SEED datasets. In this category of experiments, EEG data of six subjects were used consisting of mixing three subjects' EEG recordings from each of the DEAP and SEED datasets. Since, there are inherent differences in subject data belonging to each of the dataset, in terms of sampling rate, number and type of video clips shown to subjects, number of points recorded over time duration, and response modality (e.g., valence values (high/low) in case of DEAP and positive/negative/neutral in case of SEED). To overcome these differences in data, the data was aligned while mixing. For simplicity, we have considered only two response values from SEED data, positive and negative, this also helps in clear segregation of the mentioned classes and maps with valence level easily. The other change was that only first forty channels and 8064-point values per channel from SEED data was selected to be used for experimentation purpose. Therefore, the final size of mixed data for this category was 150x40x8064. After aligning and mixing the data, classification was done as mentioned in previous category. The results pertaining to this category are summarized in Table 3. Results suggest the effectiveness of SVM classifier for classifying this category of data. A detailed discussion on results obtained is presented in the next section.

\section{Results and Discussion}

The first category of experiments was conducted for single subject EEG data classification into respective affect classes, HV/LV for DEAP data and POS/NEG for SEED data. Table 1 summarizes classification results obtained for single subject EEG data for both datasets. The classification performance of four popular classifiers SVM, Naïve Bayes, KNN, and Tree classifiers, were assessed to select the best classifier which can be used to generalize the proposed model. The SVM classifier used in the experiments was a multiclass error-correcting output codes model which was trained on training set and then tested using twenty percent of data. The kernel function used in SVM was a polynomial kernel with order two. The classification accuracy obtained with SVM is $95 \%$ on subject belonging to DEAP data and $92.5 \%$ on subject belonging SEED dataset. A multiclass naïve Bayes model with Gaussian kernel was trained by predictors presented in trained data together with corresponding labels and classification accuracy of $87.5 \%$ was achieved after applying the proposed techniques. The third classifier used in experiments is a three neighbour KNN classifier. A fourth classifier used in our experiments is a decision tree classifier which is decision tree with binary splits for classifying data based on features and responses used to train it. Table 2 summarizes the classification results obtained for combined data of multiple subjects. In case of DEAP dataset, EEG data of ten subjects are combined and classifiers' performance are recorded.

Table 3. Accuracy (\%) for Mixed Multiple Subjects on Valence Attribute (subjects mixed from both DEAP and SEED datasets together)

\begin{tabular}{|l|c|}
\hline Classifier & Dataset \\
\hline Support Vector Machine & 92.00 \\
\hline K-Nearest Neighbor & 66.67 \\
\hline Decision Tree & 66.67 \\
\hline Naïve Bayes & 66.67 \\
\hline
\end{tabular}

Generalization is an important aspect of any real time EEG processing system and is defined to be the capability of a model or system to process any type of data. Therefore, in the next set of experiments, we tried to test the performance of chosen classifiers 
on EEG subject data mixed from two different datasets i.e., mixing data from both DEAP and SEED together. This can be considered as cross-dataset learning and we have chosen three subjects from DEAP and three subjects from SEED dataset for mixing. Then, the proposed methodology is applied, and results are recorded and presented in Table 3 . Results presented in Table 3 suggest that proposed approach is quite effective in this scenario also, and SVM classifier has shown the best classifier in terms of generalization capability. The average accuracy of SVM classifier across all cases is around $93.7 \%$ and that of Naïve Bayes classifier is $90.8 \%$. This establishes the versatility of SVM classifiers for processing EEG recordings in real time for affect categorization.

4. Model Validation

Model validation is an important step in model creation, training, and tuning. It ensures that the trained model is going to perform as expected and confirms to design objectives set. Various methods have been mentioned in the literature for performing this step. Most popular ones found in literature for accessing the performance of classifiers are cross validation, area under curve or ROC curve, and F-score. Out of these, F-score is the most robust metric as it considers class imbalances into account while accessing the performance of a classifier. In proposed work, we validated our model by computing F-scores for each category. The F-scores corresponding to each category are presented in Table 1. The proposed experimental results are also compared with the latest peer work in the same direction. Table 4 presents a thorough comparison of our work with other contemporary researchers over a single modality (valence). Analysis of figures presented in this table reveals that the proposed framework is superior to existing models for classifying EEG patterns into appropriate affect categories for a single modality valence of EEG datasets.

Table 4. Comparison with state of art research methods

\begin{tabular}{|c|c|c|}
\hline Authors & Classifier used & $\begin{array}{c}\text { Valence } \\
\text { Accuracy (\%) }\end{array}$ \\
\hline $\begin{array}{c}\text { R Majid } \\
\text { Mahboob [14] }\end{array}$ & $\begin{array}{c}\text { Deep Learning, Naïve Bayes, LDA, } \\
\text { KNN, SVM }\end{array}$ & 76.60 \\
\hline $\begin{array}{c}\text { Atkinson J. et. } \\
\text { al. [35] }\end{array}$ & $\begin{array}{c}\text { SVM (8-fold cross-validation of all } \\
\text { samples) }\end{array}$ & 73.14 \\
\hline $\begin{array}{c}\text { Mert A. et. al. } \\
\text { [36] }\end{array}$ & $\begin{array}{c}\text { ANN (leave-one-out cross- validation } \\
\text { of each subject) }\end{array}$ & 72.87 \\
\hline $\begin{array}{c}\text { Liu W. et. al. } \\
\text { [37] }\end{array}$ & $\begin{array}{c}\text { Bimodal Deep autoencoder } \\
\text { (BDAE) }\end{array}$ & $\begin{array}{c}83.25 \text { on DEAP } \\
\text { and 91.01 on } \\
\text { SEED datasets }\end{array}$ \\
\hline $\begin{array}{c}\text { Zheng W et al } \\
\text { [4] }\end{array}$ & $\begin{array}{c}\text { Discriminative Graph regularized } \\
\text { Extreme Learning Machine with } \\
\text { differential entropy features }\end{array}$ & $\begin{array}{c}69.67 \text { on DEAP } \\
\text { and 91.07 on } \\
\text { SEED datasets }\end{array}$ \\
\hline $\begin{array}{c}\text { Wichakam I. et. } \\
\text { al. [38] }\end{array}$ & $\begin{array}{c}\text { SVM (32-fold cross-validation of 32 } \\
\text { subjects) }\end{array}$ & 64.90 \\
\hline Our Work & $\begin{array}{c}\text { Four different classifiers: Naïve Bayes, } \\
\text { SVM, KNN, and Decision Tree }\end{array}$ & $\begin{array}{c}\text { 95.0 on DEEP } \\
\text { and 92.5 on } \\
\text { SEED datasets }\end{array}$ \\
\hline \multicolumn{2}{|c|}{} \\
\hline \multicolumn{2}{|c|}{}
\end{tabular}

\section{Conclusion}

This work presented a novel framework for real time processing of EEG signals for affect categorization which can be used further for automated tagging and retrieval of appropriate videos based on their emotional content and the current mood of user. The proposed approach used Generalized Procrustes analysis technique (GPA) to register the EEG recordings belonging to same category of affect modality together so that they can be classified into corresponding emotional category by the classifier. To show that the proposed method is effective thorough experiments were conducted and results were analysed to establish the stability of the model for classifying intra- as well as inter-dataset EEG patterns. Results revealed that SVM has an average accuracy of $93.7 \%$ while Naïve
Bayes is having $90.8 \%$ average accuracy. This establishes the superiority of SVM classifier and establishes its versatility for classifying almost any type of data after employing proposed method even if there are inherent differences in the data.

\section{References}

[1] S. Koelstra et al., "DEAP: A Database for Emotion Analysis; Using Physio-logical Signals," in IEEE Transactions on Affective Computing, vol. 3, no. 1, pp. 18-31, Jan.-March 2012. doi: 10.1109/T-AFFC.2011.15

[2] W.-L. Zheng, B.-L. Lu, "Investigating critical frequency bands and channels for EEG-based emotion recognition with deep neural networks", IEEE Trans. Auton. Mental Develop., vol. 7, no. 3, pp. 162-175, Sep. 2015.

[3] S. M. Alarcão and M. J. Fonseca, "Emotions Recognition Using EEG Signals: A Survey," in IEEE Transactions on Affective Computing, vol. 10, no. 3, pp. 374-393, 1 July-Sept. 2019. doi: 10.1109/TAFFC.2017.2714671

[4] W. Zheng, J. Zhu and B. Lu, "Identifying Stable Patterns over Time for Emotion Recognition from EEG," in IEEE Transactions on Affective Computing, vol. 10, no. 3, pp. 417-429, 1 July-Sept. 2019. doi: 10.1109/TAFFC.2017.2712143

[5] Preston, Simon P., and Andrew TA Wood. "Two-Sample Bootstrap Hypothesis Tests for Three-Dimensional Labelled Landmark Data." Scandinavian journal of statistics 37, no. 4 (2010): 568-587.

[6] Preston, S. P., and Andrew TA Wood. "Bootstrap inference for mean reflection shape and size-and-shape with three-dimensional landmark data." Biometrika 98, no. 1 (2011): 49-63.

[7] H. Xiong, D. Zhang, C. J. Martyniuk, V. L. Trudeau, and X. Xia, "Using generalized procrustes analysis (GPA) for normali-zation of cDNA microarray data.," BMC Bioinformatics, vol. 9, p. 25, 2008.

[8] X.-W. Wang, D. Nie, B.-L. Lu, "Emotional state classification from EEG data using machine learning approach", Neuro-computing, vol. 129, pp. 94-106, 2014.

[9] S. K. D'mello, J. Kory, "A review and meta-analysis of multimodal affect detection systems", ACM Computing. Surveys, vol. 47, no. 3, 2015.

[10] Y.-P. Lin et al., "EEG-based emotion recognition in mu-sic listening", IEEE Trans. Biomed. Eng., vol. 57, no. 7, pp. 1798-1806, Jul. 2010.

[11] M. Soleymani, J. Lichtenauer, T. Pun, M. Pantic, "A multimodal database for affect recognition and implicit tagging", IEEE Trans. Affect. Comput., vol. 3, no. 1, pp. 42-55, Jan. 2012.

[12] R. Duan, J. Zhu and B. Lu, "Differential entropy feature for EEGbased emotion classification," 2013 6th International IEEE/EMBS Conference on Neural Engineering (NER), San Diego, CA, 2013, pp. 81-84. doi: 10.1109/NER.2013.6695876

[13] R. Jenke, A. Peer and M. Buss, "Feature Extraction and Selection for Emotion Recognition from EEG," in IEEE Transactions on Affective Computing, vol. 5, no. 3, pp. 327-339, 1 July-Sept. 2014. doi: 10.1109/TAFFC.2014.2339834

[14] R. Majid Mehmood, R. Du and H. J. Lee, "Optimal Feature Selection and Deep Learning Ensembles Method for Emotion Recognition From Human Brain EEG Sensors," in IEEE Access, vol. 5, pp. 14797-14806, 2017. doi: 10.1109/ACCESS.2017.2724555

[15] D. Li et al., "The Fusion of Electroencephalography and Facial Expression for Continuous Emotion Recognition," in IEEE Access, vol. 7, pp. 155724-155736, 2019. doi: 10.1109/ACCESS.2019.2949707

[16] M.-K. Kim, M. Kim, E. Oh, S.-P. Kim, "A review on the computational methods for emotional state estimation from the human EEG", Comput. Math. Methods Med., vol. 2013, 2013. 
[17] I. B. Mauss, M. D. Robinson, "Measures of emotion: A review", Cognition Emotion, vol. 23, no. 2, pp. 209-237, 2009.

[18] Z. Lan, O. Sourina, L. Wang, Y. Liu, "Stability of features in realtime EEG-based emotion recognition algorithm", Proc. Int. Conf. Cyberworlds, pp. 137-144, Oct. 2014.

[19] A. K. Jain, R. P. W. Duin, J. Mao, "Statistical pattern recognition: A review", IEEE Trans. Pattern Anal. Mach. Intell., vol. 22, no. 1, pp. 4-37, Jan. 2000.

[20] W.-L. Zheng, B.-L. Lu, "Personalizing EEG-based affective models with transfer learning", Proc. 25th Int. Joint Conf. Artif. Intell., pp. 2732-2738, 2016.

[21] S. M. Alarcão and M. J. Fonseca, "Emotions Recognition Using EEG Signals: A Survey," in IEEE Transactions on Affective Computing, vol. 10, no. 3, pp. 374-393, 1 July-Sept. 2019. doi: 10.1109/TAFFC.2017.2714671

[22] Z. Lan, O. Sourina, L. Wang, R. Scherer and G. R. Müller-Putz, "Domain Adaptation Techniques for EEG-Based Emotion Recognition: A Comparative Study on Two Public Datasets," in IEEE Transactions on Cognitive and Developmental Systems, vol. 11, no. 1, pp. 85-94, March 2019. doi: 10.1109/TCDS.2018.2826840

[23] Gupta, Rishabh, and Tiago H. Falk. "Relevance vector classifier decision fusion and EEG graph-theoretic features for automatic affective state characterization." Neurocomputing 174 (2016): 875884.

[24] Minjie Ren, Weizhi Nie, Anan Liu, Yuting Su, "Multi-modal Correlated Network for emotion recognition in speech", Visual Informatics, Volume 3, Issue 3, 2019, Pages 150-155, ISSN 2468502X, https://doi.org/10.1016/j.visinf.2019.10.003.

[25] Fayek, Haytham M., Margaret Lech, and Lawrence Cavedon. "Evaluating deep learning architectures for Speech Emotion Recognition." Neural Networks 92 (2017): 60-68

[26] Han, Wenjing, Huabin Ruan, Xiaomin Chen, Zhixiang Wang, Haifeng Li, and Björn W. Schuller. "Towards Temporal Modelling of Categorical Speech Emotion Recognition." In Inter-speech, pp. 932-936. 2018.

[27] Liu, Zhen-Tao, Min Wu, Wei-Hua Cao, Jun-Wei Mao, Jian-Ping $\mathrm{Xu}$, and Guan-Zheng Tan. "Speech emotion recognition based on feature selection and extreme learning machine decision tree." Neurocomputing 273 (2018): 271-280.

[28] Sun, Linhui, Bo Zou, Sheng Fu, Jia Chen, and Fu Wang. "Speech emotion recognition based on DNN-decision tree SVM model." Speech Communication 115 (2019): 29-37.

[29] Hassana, Alia K., and Suhaila N. Mohammed. "A novel facial emotion recognition scheme based on graph mining." De-fence Technology (2019).

[30] He, Xuanyu, and Wei Zhang. "Emotion recognition by assisted learning with convolutional neural networks." Neuro-computing 291 (2018): 187-194.

[31] Yan, Jingwei, Wenming Zheng, Zhen Cui, Chuangao Tang, Tong Zhang, and Yuan Zong. "Multi-cue fusion for emotion recognition in the wild." Neurocomputing 309 (2018): 27-35.

[32] Y. Xu and G. Liu, "A Method of Emotion Recognition Based on ECG Signal," 2009 International Conference on Computational Intelligence and Natural Computing, Wuhan, 2009, pp. 202-205. doi: 10.1109/CINC.2009.102

[33] Guo Xianhai, "Study of Emotion Recognition Based on Electrocardiogram and RBF neural network", Procedia Engineering, Volume 15, 2011, Pages 2408-2412, ISSN 1877-7058, https://doi.org/10.1016/j.proeng.2011.08.452.

[34] Atefeh Goshvarpour, Ataollah Abbasi, Ateke Goshvarpour, "An accurate emotion recognition system using ECG and GSR signals and matching pursuit method", Biomedical Journal, Volume 40, Issue 6, 2017, Pages 355-368, ISSN 2319-4170, https://doi.org/10.1016/j.bj.2017.11.001

[35] Atkinson, J. and Campos, D., 2016. Improving BCI-based emotion recognition by combining EEG feature selection and kernel classifiers. Expert Systems with Applications, 47, pp.35-41.

[36] Mert, A. and Akan, A., 2018. Emotion recognition from EEG signals by using multivariate empirical mode decomposition. Pattern Analysis and Applications, 21(1), pp.81-89.

[37] Liu, W., Zheng, W.L. and Lu, B.L., 2016, October. Emotion recognition using multimodal deep learning. In International conference on neural information processing (pp. 521-529). Springer, Cham.

[38] Wichakam, I. and Vateekul, P., 2014, May. An evaluation of feature extraction in EEG-based emotion prediction with support vector machines. In 2014 11th international joint conference on computer science and software engineering (JCSSE) (pp. 106-110). IEEE.

[39] Tripathi, S., Acharya, S., Sharma, R.D., Mittal, S. and Bhattacharya, S., 2017, February. Using Deep and Convolutional Neural Networks for Accurate Emotion Classification on DEAP Dataset. In TwentyNinth IAAI Conference.

[40] P. Arnau-González, M. Arevalillo-Herráez, N. Ramzan (2017) Fusing highly dimensional energy and connectivity features to identify affective states from EEG signals. Neurocomputing, Volume 244, 2017, Pages 81-89, ISSN 0925-2312,

[41] Lin W., Li C., Sun S. (2017) Deep Convolutional Neural Network for Emotion Recognition Using EEG and Peripheral Physiological Signal. In: Zhao Y., Kong X., Taubman D. (eds) Image and Graphics. ICIG 2017. Lecture Notes in Computer Science, vol 10667. Springer, Cham. https://doi.org/10.1007/978-3-319-71589$6 \_33$ 